\title{
An LDR Based Colour Sensor for Urine Analysis: A Review
}

\author{
M. Chidera ${ }^{1}$, O. Kilanko ${ }^{1}$, J. Azeta ${ }^{1}$, C. A. Bolu ${ }^{1}$ \\ ${ }^{1}$ Department of Mechanical Engineering, Covenant University, Ota, Ogun State, Nigeria \\ Corresponding Author; joseph.azeta@ covenantuniversity.edu.ng
}

\begin{abstract}
The conventional way of analyzing urine colour is done by visual examination, which is often paired with urine clarity. A visual examination of urine for its colour and clarity is done to identify abnormal urine colouration, the darkness of the urine, blood in the urine, foamy urine, etc. The naked eye is used to know how clear or cloudy and the colouration of urine. It employs physical, microscopic, and chemical examination of urine. This approach is primitive and poses a great level of incompetence, which is below the present state of technology and advancement in our society. This paper gives an overview of the traditional colour sensing techniques and the use of sensors to improve colour identification accuracy. The review studies the use of various technologies such as colour sensors, smartphones, and LDR to get a better outcome in detecting urine colour using sensors and integrating artificial intelligence in the area of urine analysis.
\end{abstract}

Key words: Colour sensor, LDR, RGB, urine analysis, urine detection.

\section{INTRODUCTION}

The use of sensors has been embraced by various fields where the collection of information is a part of its operation process to prevent self-monitoring which affects the result's accuracy [1], [2]. Colour sensor identifies colour by clear index, contrast or true colour [3]. Emerging of a particular colour is a result of reflected light from an object when light falls on the object reflected, or absorbed light determines the colour observed [4]. In various applications in areas such as agriculture, medicine/health care, industry, image processing and identification, transportation, and navigation, etc. change in colour implies a change in state, operation, condition or is a requirement for the functionality of a system. Therefore, an understanding of these colours with respect to the system has been approached by different methods [5] - [7]. In a more traditional method for colour identification, the eye is used, which faces the issues of human limitation and the ability to distinguish colours, especially similar colours [8].

The eye can see a region from the light spectrum called the visible region, which makes up a certain percentage of the electromagnetic spectrum. This region of the spectrum poses a wavelength ranging from $380 \mathrm{~nm}$ to $780 \mathrm{~nm}$ and represents the ranges of colours. Light-sensitive photoreceptor cells in the human's cone cells respond most to yellow, green, and violet light with long wavelength $(570 \mathrm{~nm})$, medium wavelength $(540 \mathrm{~nm})$ and short-wavelength $(440 \mathrm{~nm})$ respectively. The nomenclature of colour identification in humans is a reaction to the wavelength of reflected light from the object. When the wavelength of a particular reflected light from the object enters the eye and strikes the retina, it triggers the associated wavelength cones (long, medium, or short) of the retina at different proportions. The trigger on the retina is detected by the brain then associate the stimulus of the light to a particular colour. Several models discussed in this review paper have been developed to extract meaning out of coloured objects and shows the relationship between primary and secondary colours.

\section{BRIEF HISTORY}

CMYK Colour Model shown in figure 1 was invented by Jacob Christoph le blon by employing secondary colour derived from opposing mixture of red, green, and blue. This secondary colour as represented in figure 1, namely cyan, magenta, and yellow while black, which is referred to as key, make the CMYK Colour Model and are used by part masking or making all CMY colours together on a white light. In converting RGB to derive CMYK, $\mathrm{C}$ is given by $1-\mathrm{R}$. M is given by $1-\mathrm{G}, \mathrm{Y}$ is given by 1 - $\mathrm{B}$ while black is an equal mixture of cyan, magenta, and yellow [9].

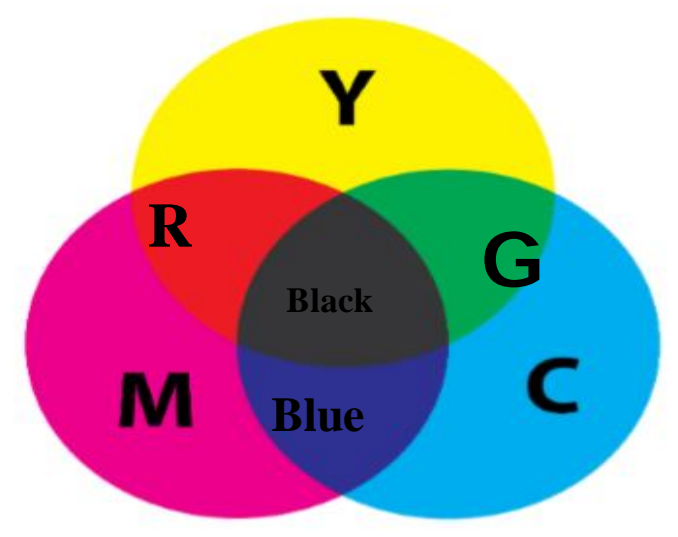

Figure 1: CMY Colour wheel [7]. 
Alvy Rau Smith in 1978 was the first to illustrate the concept of the HSV colour model as a part of a computer graphics application, which consists of hue $(\mathrm{H})$, saturation(S), and value $(\mathrm{V})$. The concept of HSV colour model depicts the makeup of colour using hue (concentration or saturation of the parent colour), saturation (shows the significant amount of white added to the colour) and value (representing the brightness and differs for $0 \%$ to $100 \%$ of added black) [10]. The grayscale consists mainly of the contrast between black and white in an image as shown in figure 2. Grayscale will not be covered as it is outside the scope of this paper. The concept of RGB Colour Mode which implies the detection and representation of colours in a system by determining the quantity of the primary colours that involves $\operatorname{red}(\mathrm{R})$, green (G) and blue (B) where the white is derived as an equal mixture of the three primary colours. The percentage of this colour segment can be ranged from $0 \%$ to $100 \%$ while the component value ranges from 0 to 255. RGB Colour Model was initiated by Thomas young and Herman Helmholtz in the theory of trichromatic-colour vision and the 19th century using James Maxwell's colour triangle. RGB Colour Model takes into account the Cartesian coordinate system as shown in figure 2. RGB based colour sensors employ the use of bayer colour filter arrays in separating the red (R), green $(G)$, and blue (B) in the input light spectrum. In obtaining frequency from light, amplification of current for input current, which precedes a current to frequency conversion is highlighted in [11]

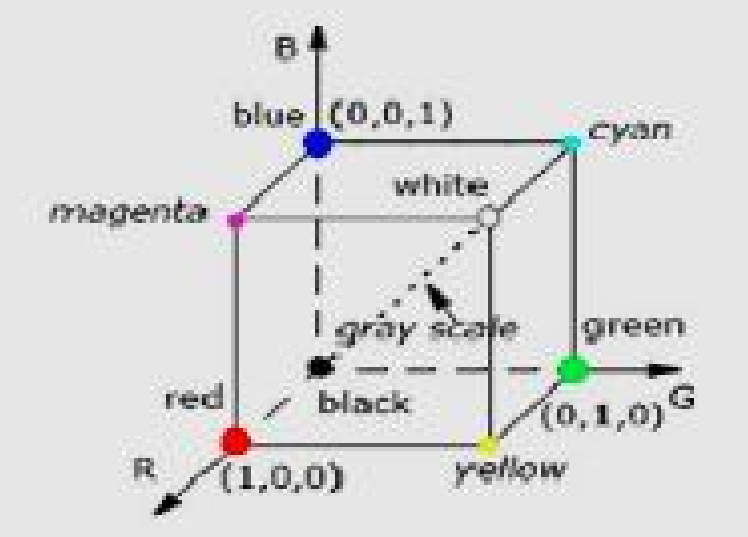

Figure 2: A schematic of RGB colour cube [9].

The colour model provides the platform that technology banks on as a technique to detect the colour of various objects having various areas of application. The RGB model provides a large percentage of the visible spectrum. The report by [12] proposed a method of detecting colour in liquid in a more accurate way by employing a TCS3200, which uses an RGB colour model, together with an STM32 microcontroller, which detects the colour of liquid across a lighted part. [13] shows the development of a concept for colour sensitivity and spectrometer free method of sensing which employs the use of plasmonic nanoholes array in conjunction with $\mathrm{L}^{*}, \mathrm{a}^{*}$, and $\mathrm{b}^{*}$ colour components. Other colour model involves CIE XYZ, CIELab, CIELUV, YUV, etc.

\subsection{Colour Sensor for Health care}

In [14], the authors employed the use of colour sensor TCS3200 and urine strip to determine the presence of albumin in the urine by the change in colour detected by the sensor with respect to the reaction of the albumin with the urine strip, which represents the amount of albumin. The TCS3200 colour sensor consists of an 8 array of a photodiode having Red, Green and Blue filters with the function of converting light energy to electrical current, then converted using a current to frequency converter to convert electrical current from the sensor to square wave frequencies and the result is obtained using an Arduino. Using the techniques above, chronic kidney disease can be detected in its early stages of kidney dysfunction and addressed. LCD instrument panel based on machine vision for colour detection involving HSI (hue, saturation, and intensity), HSV (hue, saturation, and value) and HSL (hue, saturation, and light) provided better accuracy than RGB [15].

In the area of medicine and healthcare, various changes in urine colour as a physical phenomenon can mean an abnormality [16]. Another phenomenon can change or be present to differentiate a healthy person from an unhealthy person such as specific gravity, $\mathrm{PH}$, presence of protein, nitrites, red blood cell, and white blood cell, and amount of glucose, bilirubin, and ketones. The urine at some composition or makeup is considered normal or not, which represents the health of an individual. Water is the main constituent of the urine. Other constituents involve sodium, chloride, potassium, inorganic and organic compounds, urea, and other dissolved ions. The colour of normal urine is due to its urochrome pigmentation called urobilin, which are old red cells, which form a pale yellow. Medication, food, and other diseases can also cause a change in the urine colour.

The colour of the urine can be due to fluid balance, which involves the amount of water contained in the urine that is responsible for the darkness or lightness of the urine. Diets rich in vitamin B can be supplied to the urine after the body has used up its required need, which makes the urine have a bright yellow colouration. The medication also is responsible for some urine colour such as chloroquine, iron supplements, levodopa, nitrofurantoin, phenazopyridine, phenothiazine, phenytoin, riboflavin, and triamterene, etc. [17]. In [18] several colours were highlighted as indicators to possible issues. These colours as shown in figure 3 are transparent, pale straw colour, and light orange are considered normal and healthy urine, orange urination is common as a result of an injection of certain medications such as phenazopyridine or rifampin. Brown, amber, and dark yellow are commonly associated with dehydration, which may not always be the case, brown urine colour can also be a result of rhabdomyolysis, gilbert's syndrome, or jaundice. 
Light yellow colour urine may be as a result of the presence of excess vitamin $\mathrm{B}$, maybe because of a liver or bile duct problem. Pink urination may be because of the injection of rhubarb, blueberries, and beets. Blood in the urine (hematuria), kidney stone or idiopathic, tumor, or rupture in the urinary tract or certain infection can cause red urination. An incomplete breakdown of tryptophan, which is the characteristics of blue diaper syndrome, or familial hypercalcemia which is an inherited metabolic disorder, may cause blue urine. Milky urine can be detected which may be a result of an excessive abundance of minerals such as calcium or phosphate, or protein and presence of a urinary tract infection. Phenol poison, melanoma, and copper poison lead to a dark brownish urine colouration called melanuria. Dark brown colouration can also be a result of a large intake of rhubarb, aloe, and fava beans. Green urine is a result of food and medication ingested [19].

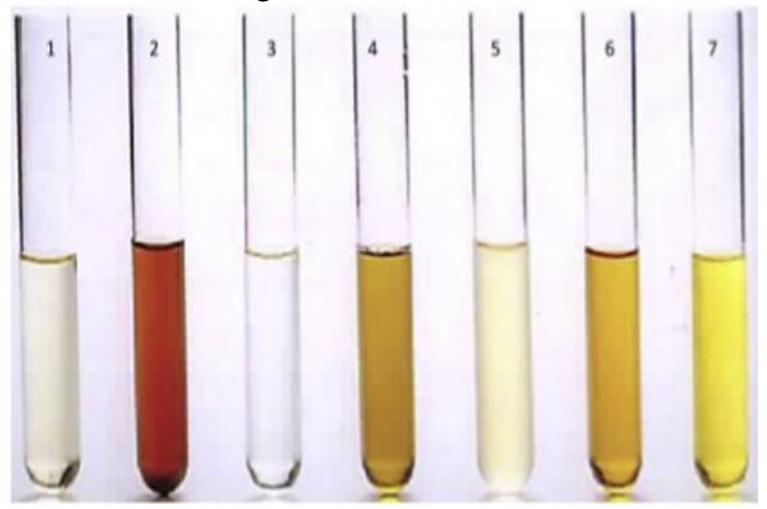

Figure 3: Different urine colour [19].

The conventional way of analyzing urine colour is done by visual examination, which is often paired with urine clarity. A visual examination of urine for its colour and clarity is done to identify abnormal urine colouration, the darkness of the urine, blood in the urine, foamy urine, etc. by this visual examination of the urine for colour and clarity is a method of urinalysis, which is an examination of urine condition [20]. The naked eye was used to know how clear or cloudy and the colouration of urine. It employs physical, microscopic, and chemical examination of urine. This approach is primitive and poses a great level of incompetence. It is also rubbishing the present state of technology and advancement in our society. A 24-hour urine collection, which is one between the common two methods of urine collection is posed with the challenges of conducting numerous manual urine analyses, which is stressful and cumbersome. The integrity of the result could be in doubt, as the detection of the colour is also a function of the soundness of the eye especially after long working hours [16].

\subsection{Colour Sensing}

A colour sensor imitates the function of the eye; it understands colour by the reaction to the intensity of light or the wavelength of light and the brain and is made up of optical and digital electronic components. A complex colour sensor system is a function of the number of wavelength band and signal channels by this, a simple three-channel colourimeter to a multi-band spectrometer can be obtained. Colour sensors work using eight colours, which are made up of primary colours and secondary colours including white, and black. A colour sensor is chosen by its desired application and is programmed to either detect colour or distinguish from multiple colour shades. The application of colour sensors is based mainly on true colour recognition and colour mark detection. True colour recognition, identifies the difference between colours and shades of a particular colour on the other hand colour mark detection identifies the change in the mask in contrast with the other mask.

The principle of colour sensing is based on the principle of optical transmission. [21] highlighted some methods of detecting the colour of a liquid as shown in the figure 4 . Three layouts were made for its optical structure in other to determine the best approach to express the colour of the liquid in a light form. The first employs the use of two lights positions at $45^{\circ}$ from the origin where the light falls on the liquid, absorption, and reflection at $90^{\circ}$. The light reflected has the colour of the liquid, which is detected. The second method employed involved the placement of the light source. The liquid sample and the colour sensor. When white light falls on the liquid, it absorbs all colour and allows the transmission of the coloured light, which is the colour of the liquid, and the sensor identifies the colour. The third, just like the first and the second employed both concept to attain the colour of a liquid. Two light sources are located at $45^{\circ}$ from the origin. The light meets at a point on a WB board and is reflected towards the liquid sample where absorption and transmission occurs. The transmitted light adopts the colour characteristics of the liquid. Due to the single light source method, the second method, which involved placing all components in a straight line, is considered as a standard method.
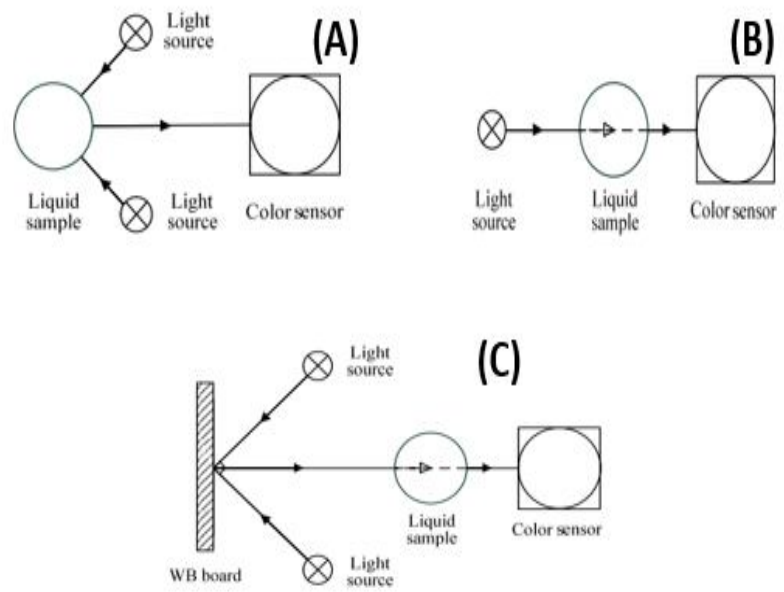

Figure 4: Three optical configurations for detection of colours in liquid [21] 
Reflective sensing theory is the principle of operation for colour sensing where detectors capture reflected light by the object, illuminant that serves as a light source whose spectrum covers the visible wavelengths and goal, which is the object whose colour is calculated, are key components in reflective sensing. To detect the colour of a goal, light is emitted on the goal by the illuminant; the light emitted on the goal is reflected in the detector. A proper detector is one with good sensitivity and spectral coverage. The spectral response should be overlapping. Mathematically, the sensor output is directly proportional to the overlapping area of the reflected and sensor spectral profile. There are two types of reflection:

- Specular reflection

- $\quad$ Diffuse reflection

The diffuse reflection is more obtainable the emitted light from the illuminant modified by the surface properties while specular reflection is characterized by a bounce off of the equal amount of light from the surface at 90 degrees with respect to the incident light hence, it carries no colour information.

A proposal for a technique was established in [11] to attain a more precise colour estimation with an optical colour sensor, which outputs a pulses series having a frequency that is proportional to the intensity of the incident light. A standardized colourimetric RGB and CIE31-XYZ colour space coordinate response were attained by applying a linear transformation in the proposed algorithm [11]. As shown in equation 1.

$\left[\begin{array}{l}X \\ Y \\ Z\end{array}\right]=\left[\begin{array}{lll}X_{R} & X_{G} & X_{B} \\ Y_{R} & Y_{G} & Y_{B} \\ Z_{R} & Z_{G} & Z_{B}\end{array}\right]=\left[\begin{array}{l}R \\ G \\ B\end{array}\right]$ Eqn 1.

Concentrations were made on optimizing the detection of colour with respect to the colour change from one RGB combination to another at a reduced response time.

\subsection{Urine Sensing Technology}

Smart approaches have been developed to reduce the contact between the urine sample and medical personnel. A programmable colour light-to-frequency converter produced by TAOS Company was employed in [22]. The converter is TCS230, which is an RGB based colour sensor for conducting urine analysis with respect to the urine colour. A summary of the major process employed by the author to detect colour involves the production of current as a response to the light reflected from the urine, the current produced is converted to voltage, which was amplified, multi-channel switch, A/D conversation and finally processed with a micro-control unit. The controlling software is illustrated by the flow chart in figure 5, which shows the processes applied by the software to detect the colour of the urine.

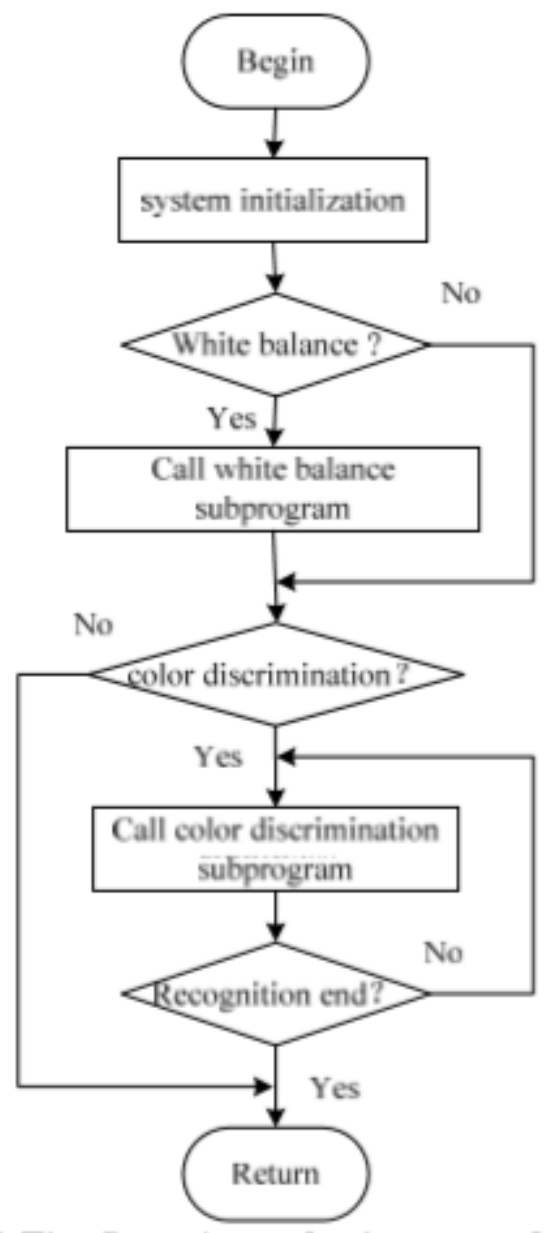

Figure 5: Flow chart of colour test software [22].

The implementation of machine learning for colourimetric analysis on test strips which was implemented by the use of an application in a smartphone. This proposition is given in [23], which poses the possibility of integrating advanced artificial intelligence in the area of urine analysis. The role of machine learning serves as a means to classify colours when sensed. The use of artificial intelligence (AI) enabling software is used to employ AI abilities while detecting colour [35]. The colour thresh-holding technique found in MATLAB is in conjunction with the RGB model to detect selected colour and can improve the impact of $\mathrm{AI}$ in colour classification as reported in [24].

The development of a urine colour system for a urine hydration system is discussed in [25]. The author employed TCS34725 as the colour sensor for the detection of urine colour that was limited to detecting the hydration state of a person daily as shown in figure 6. Support vector machines are provided as a means of classification. The system provided very accurate results when compared with urine specific gravity as a golden standard. This provided a good ground for the detection of the hydration level of an individual but a more comprehensive analysis can be obtained when considering other possible colours. 


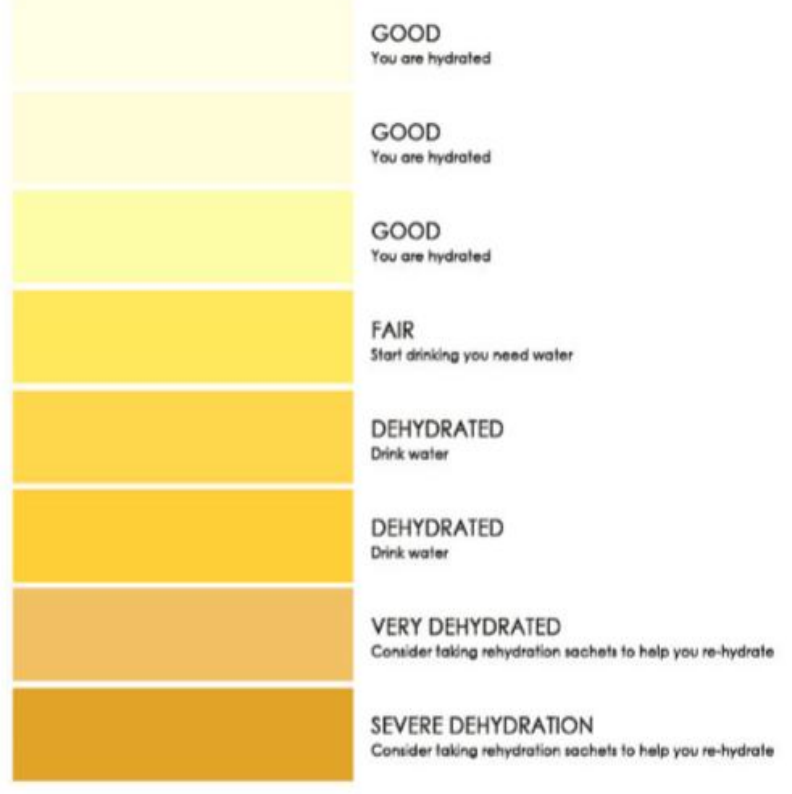

Figure 6: Colour scale for a urine hydration chart [25].

A TCS3200 colour sensor was employed in the development of a portable colour sensor based urine analysis system for the detection of chronic kidney disease. This was achieved by detecting the change in colour on a test strip due to the presence of albumin in the urine [26]. The authors designed a flowchart to describe the proposed method as shown in figure 7.

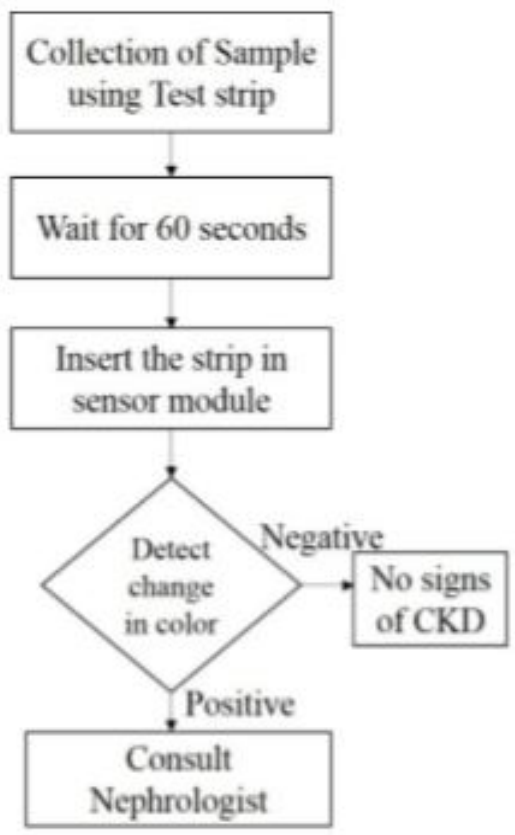

Figure 7: Operational flow chart [14].

A linear CCD was used instead of the traditional photodiode as the photoelectric converter hence improving the photoelectric conversion, which was proposed in [27]. The authors used a reflective optical system to transfer colour information from the test strip to the CCD. This system comprises an ARM microprocessor; a drive timer for the CCD drive called CPLD and is simulated using an HDL7.2 and Verilog HDL. This provided an accurate colour detection system for urine analysis. More accurate detection of the colour of liquid substances was proposed in [12] by adopting a new technique. This involves building light paths where the TCS3200 colour sensor detects colour in conjunction with an STM32 microcontroller.

The performance of colourimetric on urine using smartphones is highlighted in [28]. The challenges of using smartphones for colourimetric on urine which involves standardization was addressed by the authors. This involves the integration of colour balancing functions in smartphone cameras. In the report by [29], the authors also employed the use of smartphones in performing colourimetric analysis, discussing smartphone-based biochemical sensors, smartphone-based imaging biosensor, smartphone-based Immuno biosensor, smartphone-based hybrid biosensor as five types of smartphone-based microfluidic biosensing system at the point-of-care detection, which is applicable in urine analysis. The report in [30] investigated colourimetric biosensors, which employ the use of smartphones as detectors, and a computer analyzed the sample and sent the result back to the smartphone. The authors endeavored to break the limitations that traditional technologies for urine analysis possess on portability, easy usage, real-time monitoring, and quick response time. The immunoassay is mostly used as a sensor in a biosensor application.

\subsection{LDR Based Colour Sensor}

An LDR also called photoconductor is an electronic component or a sensor that is capable to sense the intensity of light in its environment with a response time of 8 to $12 \mathrm{~ms}$. The analog voltage produced by an LDR when exposed to light varies in magnitude which is directly proportional to the intensity of the input light. This means that the higher the intensity of the input light, the lower the resistance, and the higher the voltage while the lower the intensity of light, the higher the resistance, and the lower the voltage ranging from $0-5 \mathrm{~V}$ [31]. A more illustrative approach in [33] connotes that the working principle of an LDR comes to play when photon which is incident light that has greater energy than the energy of the LDR. This allows the movement of the electrons from the valence band to the conductive band. Hence, with increasing energy from the light, more electrons will jump to the conductive band from the valence band. This result in an increase in current allowed to flow through the sensor. Illustration from the figure 8 depicts the reaction of LDR in different light intensity using dark, daylight, and sunlight. A nonlinear relationship is observed due to the nature of the LDR. In addition, the light incident on them is of varying wavelength. 


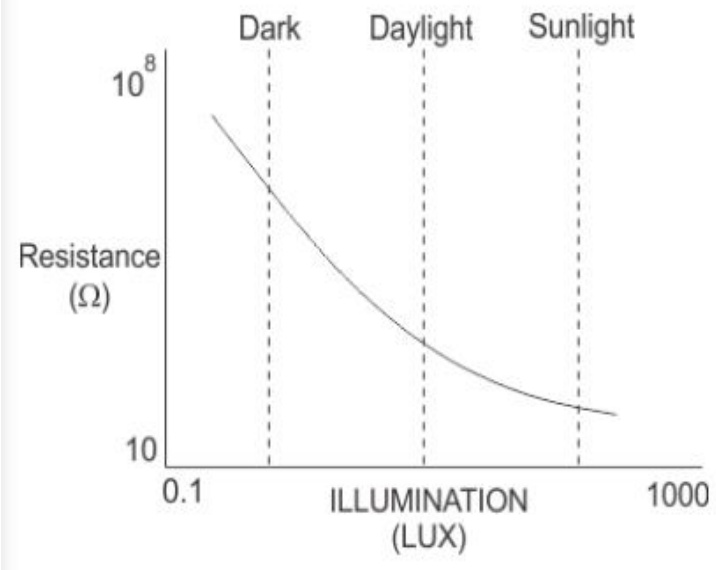

Figure 8: Graphical demonstration of the LDR's reaction to different light intensity [32]

The figure 9 shows a major component of an LDR, which includes connection pin, Cds photo-sensitive track, plated electrodes achieved by vacuum evaporation on the surface. The LDR also called a photocell can be an intrinsic photoresistor and extrinsic photoresistor where doping is the difference between both of them. Intrinsic photoresistor uses silicon or germanium which are undoped material while the extrinsic photoresistor employes the use of dopants, the dopants are impurities used to dope the photoresistor. Silicon and germanium today are replaced with cadmium sulfide and cadmium selenide lead sulfide, lead selenide, indium antimonide. The intrinsic photoresistor requires more photons to excite the electron to jump from the valence band to the conduction band while less energy is required to excite an electron to jump from the valence band to the conduction band [33].

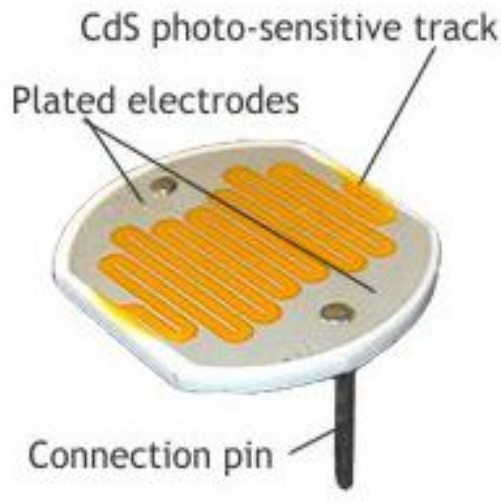

Figure 9: Components of an LDR [33].

LDR can be employed in the detection of colour owing that the principle of LDR as an electronic component operates on the varying of resistance with respect to the luminous intensity and is read by the microprocessor as employed in [34]. The authors employed the use of LDR as a colour detector for biosensing. The LDR is coupled with a red, blue, and red LED (light-emitting diode) following the RGB model. The change in resistance of the LDR due to light intensity form the three LEDs were collected by an Arduino UNO through the serial port as voltage signifying a range from 0 - 255 .

\subsection{Colour Classification and Sorting.}

Colour classification involves the sorting of results of a light wavelength reaction with a human brain or an electronic circuit. This process involves ordering and categorizing the result. While sorting, ordering involves arranging outcomes by their nature, kind, component or makeup, etc. in a particular set on the other hand categorizing involves grouping and labeling outcomes with the same attribute. Classification of colours is attained by detecting a particular colour or cluster detection in a colour space. The report in [34] highlighted that the colour classification process involves two stages. The first stage, a basic classification that uses a histogram analysis to detect colour clusters sequentially while the second stage, reclassification, involves reclassifying the result of the basic classification, which is an extracted representative colour on a colour distance. This process is represented in a flow chart shown in figure 10.

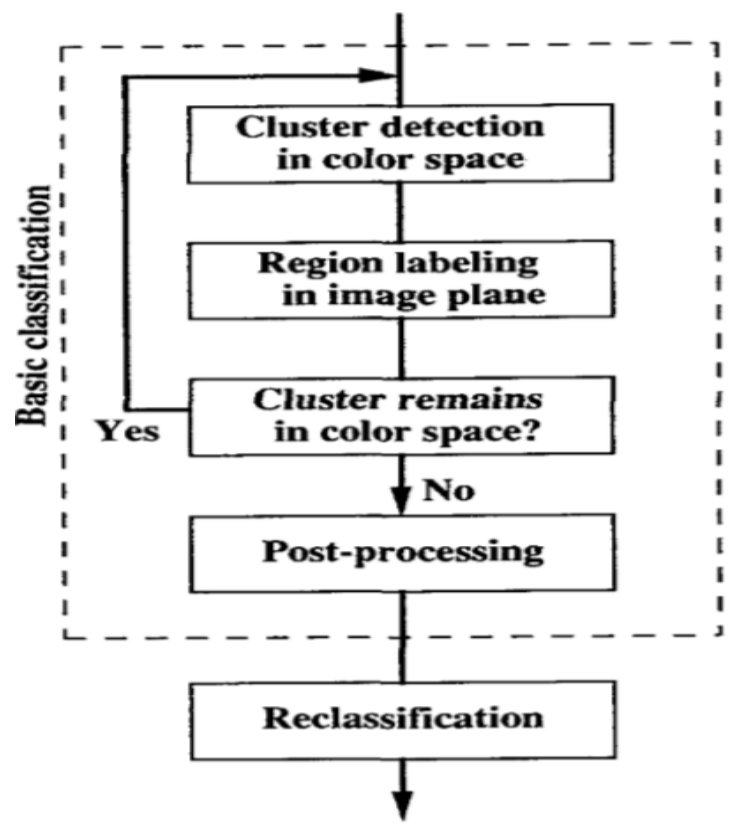

Figure 10: Processes of colour classification [34].

\section{CONCLUSION}

Colour is one of the parameters obtained while analyzing a urine sample. Traditionally, physical inspection employed to determine the colour of urine, are unreliable. The use of various methods has been developed to attain the best 
outcome when measuring a sensor. This employs the use of various technologies to get a better outcome such as detection of urine colour using sensors. The study of various seFnsing technology such as colour sensors, smartphones, and LDR were carried out. Study shows that the use of an LDR as a colour sensor produces a nonlinear colour sensor, which is based on measuring the voltage increase or drop across the circuit when incident light falls on the surface of the LDR. This results in a change in the resistance of the LDR, which in turn changes the measured voltage. The LDR was coupled with a red, blue, and red LED (light-emitting diode) following the RGB model.

\section{ACKNOWLEDGEMENT}

The authors wish to acknowledge the financial support offered by Covenant University in actualization of this research work for publication.

\section{REFERENCES}

1. Bolu, C. A., Azeta, J., Alele, F., Daranijo, E. O., Onyeubani, P., \& Abioye, A. A. (2019, December). Solar Powered Microcontroller-based Automated Irrigation System with Moisture Sensors. In Journal of Physics: Conference Series (Vol. 1378, No. 3, p. 032003). IOP Publishing.

2. Azeta, J., Bolu, C., Hinvi, D., \& Abioye, A. A. (2019, November). Obstacle detection using ultrasonic sensor for a mobile robot. In IOP Conference Series: Materials Science and Engineering (Vol. 707, No. 1, p. 012012). IOP Publishing.

3. [No title]. (n.d.-a). Retrieved April 18, 2020, from https://www.ijarse.com/images/fullpdf/1379682166_RG B_COLOUR_SENSING_TECHNIQUE.pdf

4. Colour sensor [Robotic \& Microcontroller Educational Knowledgepage - Network of Excellence]. (2018, December

http://home.roboticlab.eu/en/examples/sensor/colour

5. Gadi, V. K., Garg, A., Manogaran, I. P., Sekharan, S., \& Zhu, H.-H. (2020). Understanding Soil Surface Water Content Using Light Reflection Theory: A Novel Colour Analysis Technique Considering Variability in Light Intensity. In Journal of Testing and Evaluation (Vol. 48, Issue $\quad 5, \quad$ p. https://doi.org/10.1520/jte20180320

6. Gallinari, M. de O., de Oliveira Gallinari, M., Cintra, L. T. A., Barboza, A. C. S., da Silva, L. M. A. V., de Alcantara, S., dos Santos, P. H., Fagundes, T. C., \& Briso, A. L. F. (2020). Evaluation of the colour change and tooth sensitivity in treatments that associate violet LED with carbamide peroxide $10 \%$ : A randomized clinical trial of a split-mouth design. In Photodiagnosis and Photodynamic Therapy (Vol. 30, p. 101679). https://doi.org/10.1016/j.pdpdt.2020.101679
7. Hu, C., Mei, H., Guo, H., \& Zhu, J. (2020). Colour analysis of textile fibers by microspectrophotometry. In Forensic Chemistry (Vol. 18, p. 100221). https://doi.org/10.1016/j.forc.2020.100221

8. Zhou, Z., Di Xing, L., Qin, Y., \& Liu, L. F. (2014). Research on a Liquid Colour Detecting Device. In Advanced Materials Research (Vol. 981, pp. 602-607). https://doi.org/10.4028/www.scientific.net/amr.981.602

9. Ibraheem, N. A., Hasan, M. M., Khan, R. Z., \& Mishra, P. K. (2012). Understanding colour models: a review. ARPN Journal of science and technology, 2(3), 265-275.

10. Deswal, M., \& Sharma, N. (2014). A simplified review on fast HSV image colour and texture detection and image conversion algorithm. International Journal of Computer Science and Mobile Computing-IJCSMC, 3(5).

11. El-Hageen, H. M. M. (2020). A New Technique for Improving the Estimation of a Reflective Optical Colour Sensor. In Sensing and Imaging (Vol. 21, Issue 1). https://doi.org/10.1007/s11220-020-0276-5

12. Zhou, Z., Di Xing, L., Qin, Y., \& Liu, L. F. (2014). Research on a Liquid Colour Detecting Device. In Advanced Materials Research (Vol. 981, pp. 602-607). https://doi.org/10.4028/www.scientific.net/amr.981.602

13. Kim, S., Lee, Y., Kim, J. Y., Yang, J. H., Kwon, H.-J., Hwang, J. Y., Moon, C., \& Jang, J. E. (2019). Colour-sensitive and spectrometer-free plasmonic sensor for biosensing applications. Biosensors \& Bioelectronics, 126, 743-750.

14. Gahan, B. G., Sumir, R. M., Thomas, N., Umesh, P., Priyanka, L., Rahul, U., Chaturvedi, J., D’Souza, R., \& Tauheed, A. (2019). A Portable Colour Sensor Based Urine Analysis System to Detect Chronic Kidney Disease. In 2019 11th International Conference on Communication Systems \& Networks (COMSNETS). https://doi.org/10.1109/comsnets.2019.8711466

15. Shao-ling, D., \& Shu-guang, D. (2010). Research on the Colour Detection of LCD Instrument Panel Based on Machine Vision. In 2010 Third International Symposium on Information Science and Engineering. https://doi.org/10.1109/isise.2010.95

16. Laboratory Assessment of Kidney Disease. (2011). In Pocket Companion to Brenner and Rector's The Kidney (pp. 21-41). https://doi.org/10.1016/b978-1-4160-6640-8.00002-6

17. Urinalysis. (2019, August 13). Ucsfhealth.org. https://www.ucsfhealth.org/medical-tests/003579

18. 10 Colours That Suggest Urine Trouble. (n.d.). UC Health - UC San Diego. Retrieved May 13, 2020, from https://health.ucsd.edu/news/features/Pages/2014-04-21 -colours-that-suggest-urine-trouble.aspx

19. Callens, A. J., \& Bartges, J. W. (2015). Urinalysis. Veterinary Clinics: Small Animal Practice, 45(4), 621-637.Urinalysis: How the Test Is Done and What Results Mean | Everyday Health. (2020, April 23). EverydayHealth.com. 
M. Chidera et al., International Journal of Emerging Trends in Engineering Research, 8(10), October 2020, $7704-7711$

https://www.everydayhealth.com/urine/urinalysis-how-t est-done-what-results-mean/

20. Yetisen, A. K., Martinez-Hurtado, J. L., Garcia-Melendrez, A., da Cruz Vasconcellos, F., \& Lowe, C. R. (2014). A smartphone algorithm with inter-phone repeatability for the analysis of colourimetric tests. In Sensors and Actuators B: Chemical (Vol. 196, pp. 156-160). https://doi.org/10.1016/j.snb.2014.01.077

21. Zhou, Z., Di Xing, L., Qin, Y., \& Liu, L. F. (2014). Research on a Liquid Colour Detecting Device. In Advanced Materials Research (Vol. 981, pp. 602-607). https://doi.org/10.4028/www.scientific.net/amr.981.602

22. Solmaz, M. E., Mutlu, A. Y., Alankus, G., Kılıç, V., Bayram, A., \& Horzum, N. (2018). Quantifying colourimetric tests using a smartphone app based on machine learning classifiers. In Sensors and Actuators B: Chemical (Vol. 255, pp. 1967-1973). https://doi.org/10.1016/j.snb.2017.08.220

23. Goel, V., Singhal, S., Jain, T., \& Kole, S. (2017). Specific Colour Detection in Images using RGB Modelling in MATLAB. In International Journal of Computer Applications (Vol. 161, Issue 8, pp. 38-42). https://doi.org/10.5120/ijca2017913254

24. Gunawan, A. A. S., Brandon, D., Puspa, V. D., \& Wiweko, B. (2018). Development of Urine Hydration System Based on Urine Colour and Support Vector Machine. In Procedia Computer Science (Vol. 135, pp. 481-489). https://doi.org/10.1016/j.procs.2018.08.200

25. Zhu, L., Wang, Z., Lin, Q., \& Dong, M. (2010). Colour recognition system for urine analyzer. In Sixth International Symposium on Precision Engineering Measurements and Instrumentation. https://doi.org/10.1117/12.885816

26. [No title]. (n.d.-b). Retrieved May 19, 2020, from https://uomustansiriyah.edu.iq/media/lectures/5/5_2020 _05_03!04_28_49_AM.pdf

27. Kim, S. C., Jalal, U. M., Im, S. B., Ko, S., \& Shim, J. S. (2017). A smartphone-based optical platform for colourimetric analysis of microfluidic device. In Sensors and Actuators B: Chemical (Vol. 239, pp. 52-59). https://doi.org/10.1016/j.snb.2016.07.159.

28. Zhang, D., \& Liu, Q. (2016). Biosensors and bioelectronics on smartphone for portable biochemical detection. Biosensors \& Bioelectronics, 75, 273-284.

29. Liu, Q. X., Chen, H. Y., \& Lu, D. H. (2013). Research of Urine Analyzer Based on Colour Sensor. In Advanced Materials Research (Vol. 739, pp. 636-639). https://doi.org/10.4028/www.scientific.net/amr.739.636

30. Sanjeev, A. (2018). How to Use an LDR Sensor With Arduino.

https://maker.pro/arduino/tutorial/how-to-use-an-ldr-se nsor-with-arduino

31. Electrical4U. (2012). Light Dependent Resistor (LDR) or Photoresistor (What is it?) | Electrical4U. https://www.electrical4u.com/light-dependent-resistor-1 dr-working-principle-of-ldr/
32. Website. (n.d.). Retrieved July 19, 2020, from http://www.resistorguide.com/photoresistor/

33. Fatoni, A., Aziz, A. N., \& Anggraeni, M. D. (2020). Low-cost and real-time colour detector developments for glucose biosensor. In Sensing and Bio-Sensing Research (Vol. 28, p. https://doi.org/10.1016/j.sbsr.2020.100325

34. Tominaga, S. (n.d.). A colour classification method for colour images using a uniform colour space. In [1990] Proceedings. 10th International Conference on Pattern Recognition. https://doi.org/10.1109/icpr.1990.118220

35. Petrosov, D. A., Vashchenko, R. A., Stepovoi, A. A., \& Petrosova, N. V. (2020). Application of artificial neural networks in genetic algorithm control problems. International Journal of Emerging Trends in Engineering Research, 8(1), 177-181. 